\title{
Accesibilidad de publicación estudiantil en revistas de ciencias de la salud indizadas en SciELO
}

\author{
Carlos J. Toro-Huamanchumo, Fátima Landa-Hernández, Laura R. Arce-Villalobos, Diana Ruiz-Pingo, \\ Cristian Díaz-Vélez
}

Introducción. La investigación científica, definida como un proceso intencional de construcción de nuevo conocimiento, constituye uno de los cimientos en los cuales se sustenta la formación integral del estudiante de medicina.

Objetivo. Identificar la accesibilidad de publicación científica estudiantil en revistas de ciencias de la salud indizadas en SciELO.

Materiales y métodos. Se realizó un estudio descriptivo, transversal, cuantitativo. Se incluyeron todas las revistas de ciencias de la salud indizadas en la base de datos SciELO. Se revisaron las instrucciones para autores, se envió correo a las revistas y se realizó una búsqueda de artículos en la base de datos de cada revista. Las categorías fueron: acepta publicación estudiantil, acepta publicación estudiantil con condición, no acepta, no precisa. Los datos se fueron recogiendo en una ficha de registro.

Resultados. El 64,97\% de las revistas permite la publicación de artículos originales con estudiantes de medicina como autores. Las revistas africanas, españolas y brasileñas son las que más aceptan la publicación de estudiantes sin el requerimiento de condiciones.

Conclusión. Más de la mitad de las revistas revisadas permite la publicación estudiantil. Es importante que los alumnos de pregrado tengan conocimiento de las revistas que potencialmente podrían constituirse como cuna de sus futuras investigaciones.

Palabras clave. Estudiantes de medicina. Informe de investigación. Investigación biomédica. Publicaciones científicas y técnicas.

Sociedad Científica de Estudiantes de Medicina Veritas, SCIEMVE: Universidad de San Martín de Porres (C.J. Toro-Huamanchumo, F. LandaHernández, L.R. Arce-Villalobos,

D. Ruiz-Pingo). Oficina de Inteligencia Sanitaria; Hospital Nacional Almanzor Aguinaga Asenjo (C. Díaz-Vélez). Chiclayo, Perú.

Correspondencia: Carlos Jesús Toro Huamanchumo. El Muelle Lotes 5, 6 y 7 . Residencial Las Delicias. Dpto. 104. Urbanización Las Delicias. Chiclayo, Perú.

E-mail:

toro2993@hotmail.com

Conflicto de intereses: No declarado.

Competing interests: None declared.

(c) 2015 FEM

\section{Accessibility of student publication in health science's journals indexed in SciELO}

Introduction. Scientific research, defined as an intentional process of constructing new knowledge, is one of the foundations on which the formation of the student of medicine is based.

Aim. To identify the accessibility of student publication in journals of health sciences indexed in SciELO.

Materials and methods. A descriptive, cross-sectional and quantitative study was conducted. All the journals indexed in SciELO were included in the study. The Instructions for authors were reviewed; mails were sent to magazines and we searched for articles in the database of the journals. The categories were: accept student publication, accept student publication with condition, student publication not accepted, and not specified. The data was filled in a registration form.

Results. The $64.97 \%$ of the journals allows the publication of original articles with medical students as authors. African, Spanish and Brazilian journals are those with greater access to the publication of students without any condition.

Conclusion. More than half of the reviewed journals allow student publication. It is important that undergraduate students are aware of the journals that could potentially become the cradle of his future research.

Key words. Biomedical research. Medical students. Research report. Scientific and technical publications.

\section{Introducción}

La investigación científica, definida como un proceso intencional de construcción de nuevo conocimiento, constituye un pilar fundamental en la formación integral del estudiante de medicina, necesario para garantizar una educación médica conti- nua de calidad durante la etapa de pregrado [1,2]. No obstante, en el ámbito nacional e internacional todavía no se puede hablar de una producción científica estudiantil óptima [3,4], por lo que se requiere la implementación de estrategias de capacitación en investigación, dirigidas a los estudiantes de medicina. 
Tomando como base esta premisa, en 1986 se fundó la Federación Latinoamericana de Sociedades Científicas de Estudiantes de Medicina (FELSOCEM) [5], la cual agrupa a las distintas sociedades científicas de estudiantes de medicina, agrupaciones sin ánimos de lucro y no gubernamentales dirigidas a generar un ambiente propicio que fomente el desarrollo del espíritu investigativo en el pregrado. En Perú existe la Sociedad Científica Médico Estudiantil Peruana (SOCIMEP), que actualmente cuenta con 35 sociedades científicas estudiantiles afiliadas, todas encaminadas a la creación de un ambiente propicio para el adecuado desenvolvimiento del estudiante de medicina en el campo de la investigación [6].

Si bien existen actitudes y percepciones correctas por parte de los estudiantes acerca de formarse en una cultura de investigación [2,7], es necesario que esto se refleje no sólo en la cantidad de producción científica, sino también en su calidad, que cuente con un factor de impacto importante y no quede como una investigación más.

Hoy día se habla de un estudiante de medicina que trata de mantenerse a la vanguardia de todos los aspectos que abarca su carrera; incluso en la actualidad existen revistas científicas editadas y dirigidas por estudiantes de medicina [8]. Por esta razón, no sólo es importante fomentar la investigación en el pregrado [9], sino guiar al alumno en todo este proceso, concluyendo con la publicación de su investigación en revistas acordes con la temática de su estudio y que acepten sin restricciones la publicación estudiantil.

Al resultar necesario conocer las oportunidades existentes para que los estudiantes de pregrado publiquen sus investigaciones en revistas científicas importantes, se planteó como objetivo del estudio identificar la accesibilidad de publicación estudiantil en revistas de ciencias de la salud indizadas en SciELO.

\section{Materiales y métodos}

El presente es un estudio descriptivo, transversal, de enfoque cuantitativo. Se revisaron todas las revistas de ciencias de la salud indizadas en la base de datos SciELO (actualizado a 31 de julio de 2014). De acuerdo con el objetivo del estudio, las revistas se dividieron en las siguientes categorías:

- Acepta publicación estudiantil.

- Acepta publicación estudiantil con condiciones.

- No acepta publicaciones de estudiantes.

- No precisa.
El estudio se dividió en varias etapas. La primera consistió en la revisión de todas las instrucciones para autores' de las revistas y en completar la ficha de registro de acuerdo con las categorías anteriores. Si la revista no precisaba si aceptaba o no la publicación de estudiantes, se pasaba a la segunda etapa, en la cual se envió un correo dirigido al editor en jefe o a secretaría editorial de la revista. Se dio un plazo de 30 días hábiles para la obtención de respuesta y, paralelamente, se fue cumplimentando la ficha de registro. Finalmente, en la tercera etapa se incluyeron aquellas revistas que no respondieron al correo mediante un análisis independiente donde se buscó, en su base de datos, la presencia de artículos con estudiantes de medicina como autores, registrando los hallazgos en una categoría aparte.

Se utilizaron los programas Excel 2010 y SPSS v. 19.0 para el registro y análisis descriptivo de los datos. Se calcularon frecuencias y se obtuvo una presentación tabulada de los resultados.

\section{Resultados}

Se revisaron todas las revistas de ciencias de la salud indizadas en SciELO (hasta el 31 de julio de 2014), constituyendo un total de 294 revistas distribuidas en 12 países. En la primera fase del estudio sólo se obtuvieron datos de 22 revistas; en la segunda, correspondiente al envío de correos, se obtuvieron los datos de 196 más, quedando finalmente 76 revistas que entraron en la tercera fase del estudio (Figura).

Los países con mayor número de revistas de ciencias de la salud indizadas en SciELO fueron: Brasil ( $n=93 ; 31,63 \%)$, Colombia $(n=39 ; 13,27 \%)$, España $(n=38 ; 12,93 \%)$ y Cuba $(n=34 ; 11,57 \%)$ (Tabla I). Se encontró que el $64,97 \%$ de las revistas permite la publicación de artículos originales con estudiantes de medicina como autores: el 22,45\% anexa alguna condición, como el hecho de que haya un médico o profesional de ciencias de la salud como autor principal o coautor de la investigación, y el 42,52\% permite libremente la publicación estudiantil sin condición alguna (exceptuando naturalmente su calidad y el cumplimiento de las normas para autores y del proceso editorial). El 9,18\% de las revistas no aceptan la publicación de estudiantes y 76 (25,85\%) no precisan esta información.

Cuba fue el país con mayor porcentaje de revistas $(29,41 \%)$ que no acepta artículos originales con estudiantes como autores. El 50\% de las revistas costarricenses de ciencias de la salud tampoco aceptan publicación estudiantil, aunque el país sólo cuenta con cuatro revistas indizadas. Las revistas africanas, 
Tabla I. Países con revistas de ciencias de la salud indizadas en SciELO $(n=294)$.

\begin{tabular}{ll}
\hline Argentina & $15(5,10 \%)$ \\
\hline Brasil & $93(31,63 \%)$ \\
\hline Chile & $17(5,78 \%)$ \\
\hline Costambia & $39(13,27 \%)$ \\
\hline Cuba & $4(1,36 \%)$ \\
\hline España & $34(11,57 \%)$ \\
\hline México & $38(12,93 \%)$ \\
\hline Perú & $11(3,74 \%)$ \\
\hline Portugal & $7(2,38 \%)$ \\
\hline Sudáfrica & $14(4,76 \%)$ \\
\hline Venezuela & $8(2,72 \%)$ \\
\hline
\end{tabular}

argentinas, españolas y brasileñas son las que más aceptan publicación de estudiantes sin solicitar condición alguna $(75 \%, 53,33 \%, 47,37 \%$ y 47,31\%, respectivamente) (Tabla II).

Sudáfrica es el país con mayor accesibilidad a la publicación de artículos originales de estudiantes de medicina, con un total de seis de sus ocho revistas de ciencias de la salud indizadas. Perú y Venezuela le siguen con un $71,43 \%$ y $71,41 \%$ de sus revistas, respectivamente; aunque el primero sólo tiene hasta la fecha cinco revistas indizadas (Tabla III).

\section{Discusión}

De las 294 revistas, en la primera fase del estudio sólo pudieron obtenerse datos concisos sobre la accesibilidad de publicación estudiantil en 22 de ellas, lo cual es un indicador de la falta de información y precisión en las 'instrucciones para autores' de las revistas. Ello podría ser una limitación para el envío de artículos por parte de los estudiantes, al suponer erróneamente, en algunos casos, que no se permite bajo ninguna circunstancia la publicación de artículos que sean de su autoría.

Hubo un considerable número de respuestas a los correos que se enviaron, lo cual sugiere la presencia de comités editoriales activos, disponibles y
Figura. Representación esquemática del proceso de selección y evaluación de las revistas incluidas en el estudio.

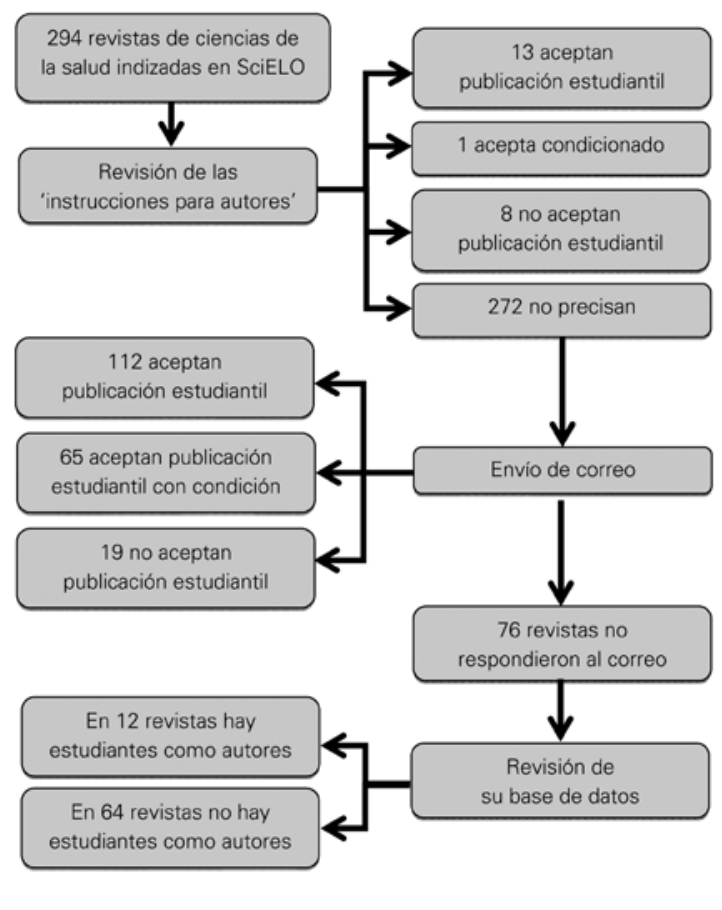

atentos a solventar en breve plazo las dudas que puedan tener los autores. Esto debe destacarse porque actualmente las revistas de mayor prestigio se precian de fijar plazos relativamente breves para responder las inquietudes de los autores y cumplimentar las diferentes etapas del proceso editorial, lo cual constituye un indicador esencial de la calidad de dicho proceso [10].

Brasil indudablemente aventaja al resto de países no sólo por el hecho de tener un mayor número de revistas de ciencias de la salud indizadas en SciELO, sino por ser el país con mayor número de revistas en general incluidas en esta base de datos [11]. Además, más de la mitad de sus revistas permite la publicación de artículos con estudiantes como autores, lo cual repercute también en la producción científica del país, uno de los líderes en los rankings de investigaciones científicas [12]. En Perú es necesario seguir trabajando para mejorar la visibilidad e impacto de las revistas médicas, pues pocas se encuentran indizadas en diferentes bases de datos [13].

Un dato interesante es que más de la mitad de revistas permite la publicación estudiantil, ya sea condicionada o no. Entonces, ¿por qué existiendo 
Tabla II. Accesibilidad de publicación estudiantil en las revistas de ciencias de la salud indizadas en SciELO, según el país (porcentaje calculado sobre el total por fila).

\begin{tabular}{lcccc}
\hline & $\begin{array}{c}\text { Revistas que } \\
\text { aceptan }\end{array}$ & $\begin{array}{c}\text { Revistas que aceptan } \\
\text { condicionado }\end{array}$ & $\begin{array}{c}\text { Revistas que } \\
\text { no aceptan }\end{array}$ & $\begin{array}{c}\text { Revistas que } \\
\text { no precisan }\end{array}$ \\
\hline Argentina & $8(53,33 \%)$ & $2(13,33 \%)$ & $1(6,67 \%)$ & $4(26,67 \%)$ \\
\hline Brasil & $44(47,31 \%)$ & $16(17,20 \%)$ & $1(1,08 \%)$ & $32(34,41 \%)$ \\
\hline Colomile & $7(41,18 \%)$ & $5(29,41 \%)$ & 0 & $5(29,41 \%)$ \\
\hline Costa Rica & $1(25,00 \%)$ & $1(25,00 \%)$ & $2(50,00 \%)$ & $7(17,95 \%)$ \\
\hline España & $18(47,37 \%)$ & $6(15,79 \%)$ & $4(10,53 \%)$ & $10(26,32 \%)$ \\
\hline México & $5(45,45 \%)$ & $1(9,09 \%)$ & 0 & $5(45,45 \%)$ \\
\hline Perú & $3(42,86 \%)$ & $2(28,57 \%)$ & 0 & $2(28,57 \%)$ \\
\hline Portugal & $6(42,86 \%)$ & $2(14,29 \%)$ & $3(21,43 \%)$ & $3(21,43 \%)$ \\
\hline Sudáfrica & $6(75,00 \%)$ & 0 & 0 & $2(25,00 \%)$ \\
\hline Venezuela & $5(35,71 \%)$ & $5(35,71 \%)$ & 0 & $4(28,57 \%)$ \\
\hline
\end{tabular}

oportunidades de publicar en revistas internacionales aún parecen haber limitaciones para que la producción y la publicación científica de los estudiantes aumenten realmente de manera considerable? Esto sería un indicador de que no hablamos de falta de oportunidades, sino de iniciativa por parte de los estudiantes [14].

$\mathrm{Si}$ bien en los últimos años se han observado mejoras en la producción científica estudiantil, tanto nacional [15] como internacional [16], esta situación todavía no se corresponde con las oportunidades para publicar que ofrecen las revistas. Ello puede deberse a que comúnmente se cometen errores en la redacción del artículo [17], lo cual representa una limitación para su aceptación en revistas internacionales, que presentan una rigurosa evaluación por pares y una tasa de rechazo acorde con su prestigio [18]. A ello se suma la falta de capacitación en métodos de investigación que, junto con la falta de tiempo, constituyen los principales obstáculos para la publicación científica estudiantil $[19,20]$.

La existencia de revistas que no aceptan la publicación de estudiantes puede entenderse por el hecho de ser publicaciones oficiales de sociedades médicas que aceptan únicamente aportaciones de especialistas en el área de la revista. No obstante, una
Tabla III. Accesibilidad de publicación estudiantil con o sin condición, según el país $(n=191)$.

\begin{tabular}{ll}
\hline Argentina & $10(66,66 \%)$ \\
\hline Brasil & $60(64,51 \%)$ \\
\hline Colombia & $12(70,59 \%)$ \\
\hline Costa Rica & $26(66,67 \%)$ \\
\hline Cuba & $2(50,00 \%)$ \\
\hline España & $22(64,71 \%)$ \\
\hline México & $24(63,16 \%)$ \\
\hline Perú & $6(54,54 \%)$ \\
\hline Portugal & $5(71,43 \%)$ \\
\hline Sudáfrica & $8(57,15 \%)$ \\
\hline Venezuela & $6(75,00 \%)$ \\
\hline
\end{tabular}

alternativa que no debería descartarse es permitir la publicación de estudiantes como coautores, pues actualmente algunas escuelas médicas realmente hacen hincapié en la incorporación del método científico en la práctica clínica y la educación básica de sus estudiantes, lo cual brinda un nivel de conocimiento adecuado que se refleja de manera positiva en el proceso de investigación científica [8].

En conclusión, existe un buen número de revistas de ciencias de la salud indizadas en SciELO que aceptan la publicación de estudiantes de medicina.

Es importante que los alumnos de pregrado tengan conocimiento de las revistas que potencialmente podrían constituirse como cuna de sus investigaciones. Estudios futuros deben evaluar la aportación estudiantil anual en estas revistas, verificando que exista un aumento progresivo de las publicaciones. Del mismo modo, deben realizarse estudios similares usando las bases de datos más reconocidas mundialmente en el ámbito de ciencias de la salud: Medline, ISI y SCOPUS.

Bibliografía

1. Cabrera-Enríquez JA, Cruzado-Mendoza C, Purizaca-Rosillo N, López-Samanamú RO, Lajo-Aurazo Y, Peña-Sánchez ER, et al. Factores asociados con el nivel de conocimientos y la actitud hacia la investigación en estudiantes de medicina en Perú, 2011. Rev Panam Salud Pública 2013; 33: 166-73. 
2. Mayta-Tristán P, Cartagena-Klein R, Pereyra-Elías R, Portillo A, Rodríguez-Morales AJ. Apreciación de estudiantes de medicina latinoamericanos sobre la capacitación universitaria en investigación científica. Rev Med Chile 2013; 141: 716-22.

3. Taype-Rondán A, Huaccho-Rojas J, Guzmán L. Sociedades científicas de estudiantes de medicina en el Perú: situación actual y perspectivas futuras. CIMEL 2011; 16: 90-5.

4. Nel D, Burman RJ, Hoffman R, Randera-Rees S. The attitudes of medical students to research. S Afr Med J 2014; 104: 32-6.

5. Mondragón-Cardona A, Jiménez-Canizales CE, AlzateCarvajal V. Oportunidades y desarrollo en las sociedades científicas estudiantiles. CIMEL 2012; 17: 51.

6. Toro-Huamanchumo CJ. ¿Por qué pertenecer a sociedades científicas en el pregrado de medicina? Rev Cuerpo Med HNAAA 2014; 7: 43-4.

7. Carrillo-Larco RM, Carnero AM. Autoevaluación de habilidades investigativas e intención de dedicarse a la investigación en estudiantes de primer año de medicina de una universidad privada en Lima, Perú. Rev Med Hered 2013; 24: 17.

8. Cabrera-Samith I, Oróstegui-Pinilla D, Ángulo-Bazán Y, Mayta-Tristán P, Rodríguez-Morales AJ. Revistas científicas de estudiantes de medicina en Latinoamérica. Rev Med Chile 2010; $138:$ 1451-5.

9. Arráez-Aybar LA, Millán Núñez-Cortés J, Carabantes-Alarcón D, Lozano-Fernández R, Iglesias-Peinado I, Palacios-Alaiz E, et al. Adquisición de competencias transversales en alumnos de pregrado de Ciencias de la Salud en la Universidad Complutense: una experiencia positiva. Educ Med 2008; 11: 169-77.

10. Alfonso-Manzanet JE, Silva-Ayçaguer LC. Gestión automatizada en el proceso editorial de una revista científica como demanda inaplazable para favorecer la cultura comunicacional. Educ Med Super 2014; 28: 145-53.

11. Miguel S. Journals and scientific production in Latin America and the Caribbean: its visibility in SciELO, RedALyC and SCOPUS. Rev Interam Bibliotecol 2011; 34: 187-98.

12. Marziale MHP. Indicators of Ibero-American scientific production. Rev Lat Am Enfermagem 2011; 19: 853-4.

13. Soriano-Álvarez C. Investigación científico-médica en el Perú: nuevos espacios. Rev Gastroenterol Perú 2013; 33: 207-8.

14. Mabvuure NT. Low medical student publishing rates: lack or opportunity or initiative? Med Teach 2012; 34: 254-5.

15. Taype-Rondán A, Lajo-Aurazo Y, Gutiérrez-Brown R, ZamalloaMasías N, Saldaña-Gonzales M. Aporte de las sociedades estudiantiles en la publicación científica en Scielo-Perú, 20092010. Rev Peru Med Exp Salud Publica 2011; 28: 691-2.

16. Wickramasinghe DP, Perera CS, Senarathna S, Samarasekera DN Patterns and trends of medical student research. BMC Med Educ 2013; 13: 175.

17. Franco C, Rodríguez-Morales AJ. Errores comunes en la redacción científica estudiantil. Gac Med Caracas 2010; 118: 69-72.

18. Stahel PF, Moore EE. Peer review for biomedical publications: we can improve the system. BMC Med 2014; 12: 179.

19. Griffin MF, Hindocha S. Publication practices of medical students at British medical schools: experience, attitudes and barriers to publish. Med Teach 2010; 33: e1-8.

20. Alsayed N, Eldeek B, Tayeb S, Ayuob N, Al-Harbi A. Research practices and publication obstacles among interns at King Abdulaziz University Hospital, Jeddah, Saudi Arabia, 2011 2012. J Egypt Public Health Assoc 2012; 87: 64-70. 